the three treatment groups. However, chlorthalidone was superior to amlodipine in the 6-year rate of heart failure (HF), $7.7 \%$ vs. $10.2 \%$, relative risk 1.38 , and chlorthalidone was superior to lisinopril in the 6-year rates of combined cardiovascular disease, $30.9 \%$ versus $33.3 \%$, RR 1.10 , stroke (5.6\% versus $6.3 \%$, RR 1.15 ) and $\mathrm{HF}$ (8.7\% versus $7.7 \%$, RR 1.19). Robert Anderson, one of the ALLHAT investigators, has additional observations regarding the ALLHAT study findings in this issue of the Journal. ${ }^{65}$

\section{Prior Authorization to Manage Drug Utilization and Costs}

In 1998, state Medicaid programs were responding in various ways to double-digit increases in prescription drug benefit costs. In some states such as Massachusetts, the cost increases threatened to push drug benefit costs ahead of the costs of hospital acute care services by the year 2003. Massachusetts Medicaid planned to implement "more aggressive utilization practices," Kentucky required prescribers to use lower-cost first-generation antihistamines and Florida used prescriber profiling, focusing on the use of "fourth-generation" antibiotics for upper respiratory infection in adults. ${ }^{66}$ Florida also planned "provider education forums" to focus on drug prices. Drug formularies were not an option since state Medicaid programs have essentially open formularies because nearly all drug manufacturers pay rebates to obtain formulary status, leaving only prior authorization (PA) programs to discourage use of certain high-cost drugs. Medicaid rebates for single source and innovator multiple-source brand drugs in 1998 was the greater of $15.1 \%$ of average manufacturer price or AMP less best price. For noninnovator drugs, the rebate was $11 \%$.

By 1999 and 2000, state Medicaid officials began to complain more openly about requirement of state programs to operate open drug formularies as part of the OBRA 1990 statute on mandatory drug manufacturer rebates. Oklahoma Health Care Authority CEO, Michael Fogarty, testified at a 29 March 2000 Senate Finance Committee hearing that two factors in the Medicaid "best price" approach contributed to the "evaporation" of savings: (a) open formularies, and (b) price adjustments by manufacturers to compensate for the mandatory rebates. ${ }^{67}$ Fogarty recommended that state Medicaid programs be permitted to institute closed formularies to make drug manufacturers compete with lower prices.

OBRA 1993 amended the OBRA 1990 language and no longer required state Medicaid programs to reimburse for new drugs approved by the FDA, for the first 6 months after introduction. However, federal law and regulations prohibit states from denying access to drugs by Medicaid recipients, and 43 states and the District of Columbia had PA programs in place in 1996 to limit the use of nonformulary and nonpreferred drugs. ${ }^{68}$ States have reported limited success with PA programs, and New York adopted a mandatory generic drug substitution program in November 2002 in which brand-name drugs with a generic equivalent would require a PA to be covered by the Medicaid program. The PA program could be utilized by physi- cians using voice recognition or a keypad and required the physicians to answer a "brief set of questions about why the patient required the brand product." For a multiple-source brand product to be dispensed, the prescription must include the PA number obtained by the physician, and must indicate "DAW" (dispense as written) and "brand (medically) necessary." York hoped to push its generic substitution rate to 95\% from $88 \%$ of multiple-source brand drug prescriptions with the mandatory generic/PA program, a modest goal for managed care organizations working in the private employer sector.

The absence of reliable data on the cost-effectiveness of prescription drug benefit prior PA programs was highlighted in a previous issue of the Journal. ${ }^{70}$ Any reasonable assessment of the cost-effectiveness of PA programs in prescription drug benefits would include consideration of the potential, and predictable, adverse service outcomes, including physician, pharmacy, and beneficiary, satisfaction as well as wait time and additional service costs. Such considerations in the public arena appear to be outweighed by budget concerns. Beginning with the Florida PA program for nonpreferred drugs that was launched in July 2001, several other states implemented similar programs by mid-2002. ${ }^{71}$ The principal feature of all of these state Medicaid PA programs, including Michigan, Illinois, Louisiana, and North Carolina, was to extract additional rebates from pharmaceutical manufacturers to bring their drugs down to the "reference price" within therapeutic classes. Failure to match the reference price through supplemental rebates subjects the drug to PA. Cox- 2 drugs were the first of 2 drug classes implemented in the Louisiana Medicaid preferred drug program in June 2002.

In this issue of the Journal, LaPensee describes the experience of a drug PA program in a Medicaid managed care organization (MCO) in the northeast in early 2002. ${ }^{72}$ While Medicaid HMOs operate under a different set of rules than private MCOs and have fewer tools to manage drug benefit costs, particularly the absence of tier-copay benefit designs, the description of this Medicaid PA program has some useful information for all managed care observers. Nearly 4 out of 5 PA requests were for formulary drugs. Second, the PA rejection rate was low: only $4.4 \%$ of the more than 22,000 PA requests received each month were denied. Clearly, this is a large administrative burden, nearly 1,000 PA requests per day, of which an average of 44 were denied. The 93\% acceptance rate for PAs for nonformulary drugs compares with an acceptance rate of about $75 \%$ for drug benefit PAs for commercial health plans in 1999 and 2000..$^{\text {73-74 }}$

\section{HIPAA Effects on Health Research and PBM Functions in Drug Utilization Review}

The Health Insurance Portability and Accountability Act (HIPAA) of 1996 was crafted originally with the intent to better protect health insurance coverage for employees and their families when employees changed employers; i.e., to ensure portability of health insurance. ${ }^{75}$ By the time the legislative process was complete, HIPAA had 2 primary impacts beyond portabil- 